\title{
Crack growth path prediction for the angled cracked plate using higher order terms of Williams series expansion
}

\author{
S. Nasiraldin Mirlohi ${ }^{a^{*}}$ and M.R.M. Aliha ${ }^{b}$
}

\begin{abstract}
${ }^{a}$ Department of Mechanical Engineering and Projects, Solaris-mci EPCM in Oil \&Gas Surrey, BC, Canada
${ }^{b}$ Welding and Joining Research Center, Iran University of Science and Technology, Narmak, 16846-13114, Tehran, Iran

A R T ICLE INFO A B S T RACT

Article history:

Received March 20, 2013

Received in Revised form

September, 14, 2013

Accepted 18 September 2013

Available online

18 September 2013

Keywords:

Williams series

Crack growth path

Mixed Mode

Maximum tangential stress
\end{abstract}

criterion

Higher order terms

\section{Introduction}

Determination of the direction and the path of crack growth can play an important role for predicting the amount of damage induced by brittle fracture in the structures or engineering components. In the case of mixed mode loading I/II (combined opening and in-plane sliding deformation), crack growth does not grow along the direction of initial crack and the fracture trajectory in general is a curvilinear path. Several studies have been completed to estimate the initial crack growth direction under mixed mode (Aliha \& Ayatollahi, 2009, 2012, Ayatollahi \& Aliha 2009, Aliha et al. 2010, Ayatollahi et al. 2011), but little research studies have been performed for estimating the mixed mode crack growth path. Studies to determine the crack growth path have led to two main methods (Leevers et al. 1976, Maiti \& Prasad 1980, Alpa et al. 1980, Maiti \& Smith 1983, Sumi 1985): (1) Incremental crack growth method and (2) the fracture threshold method. In the first method, the gradual crack growth is

* Corresponding author.

E-mail addresses: n.mirlohi@gmail.com(S. Nasiraldin Mirlohi)

(C) 2013 Growing Science Ltd. All rights reserved.

doi: $10.5267 / \mathrm{j}$.esm. 2013.09 .003 
modeled based on the one of the critical mechanical parameters, such as the strain energy density, strain energy release rate or stress intensity factor. This method involves a large number of small crack extensions in appropriate directions. The direction of crack growth for each increment can be determined by means of the available mixed mode fracture theories such as the maximum tangential stress (Erdogan \& Sih, 1963), the minimum strain energy density criterion (Sih 1974), the maximum energy release rate criterion (Hussain et al. 1974), and the cohesive zone model (Gomez et al. 2009). Accordingly, a certain increment of crack growth is chosen and the crack geometry and direction is modified in each increment using one of the mentioned criteria until the final fracture path. Due to the continuous deformation of a crack relative to its initial shape, the use of numerical analyses such as finite element method is often necessary for modeling the trajectory of growing cracks. For example, Ayatollahi et al. (2006), Aliha et al. (2010, 2012) and Aliha and Rezaei (2011) determined numerically mixed mode fracture path of some test specimens using the finite element simulations by means of the incremental crack growth method.. However In the second method (i.e the fracture threshold method), regardless of any progressive growth of the crack, the crack growth path is estimated based on the distribution of mechanical parameters such as stress, strain or similar parameters at the onset of failure.

In this paper, the path of mixed mode fracture growth in a well-known cracked test specimen (called the angled center crack plate) is investigated theoretically and for predicting the path of fracture the maximum tangential stress (MTS) criterion is used. For this purpose, first the distribution of maximum tangential stress should be obtained for the given cracked component using either numerical or analytical methods. In the next section following a brief description of the specimen, the Williams series expansion for the crack tip stresses is also outlined. It is shown that the number of terms considered in the Williams series expansion has a significant influence on the fracture trajectory estimated by the MTS criterion.

\section{Crack tip stresses for the angled center crack plate}

The crack growth in a sheet with a central angle crack and subjected to uniaxial or biaxial far field loads (shown in Figure 1) is a typical example of mixed-mode fracture which has been investigated by many researchers in the past. This problem was investigated for the first time by Erdogan and Sih (1963) where the MTS was applied to predict the initial direction of crack propagation and also the critical load of body at the onset of fracture. Based on the MTS criterion, the crack will grow radialy along the direction of maximum tangential stress $\theta_{c}$. The crack growth occurs when the maximum value of tangential stress component $\sigma_{\theta \theta}$ reaches a critical value, $\sigma_{\theta \theta c}$ which is assumed as a constant material property.

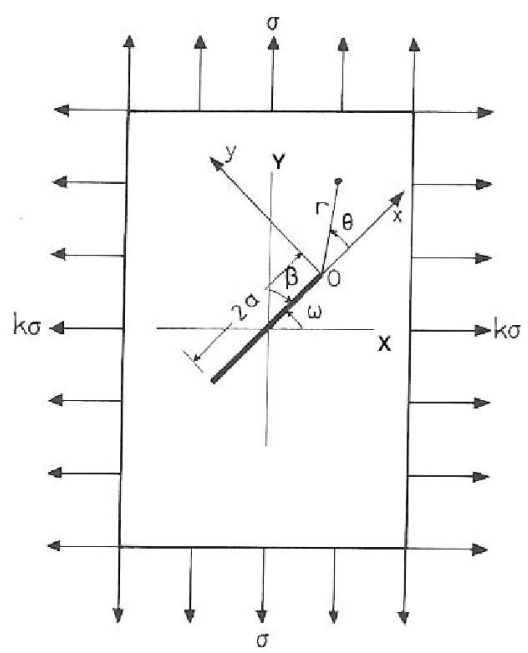

Fig. 1. The angled crack plate subjected to far field biaxial loading 
The stress field around the crack tip for a center cracked body subjected to far field stress $\sigma_{\infty}$ can be written by Williams (1957) series expansion as (Papadopoulos, 1993):

$$
\begin{aligned}
& \sigma_{x}=\sigma_{\infty}(1-k) \cos 2 \beta+\frac{K_{I}}{2 \sqrt{\pi a}}\left((r / 2 a)^{-1 / 2} \cos \frac{\theta}{2}\left(1-\sin \frac{\theta}{2} \sin \frac{3 \theta}{2}\right)+\right. \\
& \left.\frac{3}{2}(r / 2 a)^{1 / 2} \cos \frac{\theta}{2}\left(1+\sin ^{2} \frac{\theta}{2}\right)+\sum_{n=1}^{\infty}(r / 2 a)^{n+\frac{1}{2}} C_{n}\left[\cos \left(n+\frac{1}{2}\right) \theta-\left(n+\frac{1}{2}\right) \sin \theta \sin \left(n-\frac{1}{2}\right) \theta\right]\right) \\
& +\frac{K_{I I}}{2 \sqrt{\pi a}}\left(-(r / 2 a)^{\frac{-1}{2}} \sin \frac{\theta}{2}\left(2+\cos \frac{\theta}{2} \cos \frac{3 \theta}{2}\right)+\frac{3}{2}(r / 2 a)^{\frac{1}{2}} \sin \frac{\theta}{2}\left(2+\cos ^{2} \frac{\theta}{2}\right)+\right. \\
& \sum_{n=1}^{\infty}(r / 2 a)^{n+\frac{1}{2}} C_{n}\left[2 \sin \left(n+\frac{1}{2}\right) \theta+\left(n+\frac{1}{2}\right) \sin \theta \cos \left(n-\frac{1}{2}\right) \theta\right] \\
& \sigma_{y}=\frac{K_{I}}{2 \sqrt{\pi a}}\left((r / 2 a)^{-1 / 2} \cos \frac{\theta}{2}\left(1+\sin \frac{\theta}{2} \sin \frac{3 \theta}{2}\right)+\right. \\
& \left.\frac{3}{2}(r / 2 a)^{1 / 2} \cos ^{3} \frac{\theta}{2}+\sum_{n=1}^{\infty}(r / 2 a)^{n+\frac{1}{2}} C_{n}\left[\cos \left(n+\frac{1}{2}\right) \theta+\left(n+\frac{1}{2}\right) \sin \theta \sin \left(n-\frac{1}{2}\right) \theta\right]\right) \\
& +\frac{K_{I I}}{2 \sqrt{\pi a}}\left((r / 2 a)^{\frac{-1}{2}} \sin \frac{\theta}{2} \cos \frac{\theta}{2} \cos \frac{3 \theta}{2}-\frac{3}{2}(r / 2 a)^{\frac{1}{2}} \sin \frac{\theta}{2} \cos ^{2} \frac{\theta}{2}-\right. \\
& \sum_{n=1}^{\infty}(r / 2 a)^{n+\frac{1}{2}} C_{n}\left[\left(n+\frac{1}{2}\right) \sin \theta \cos \left(n-\frac{1}{2}\right) \theta\right] \\
& \tau_{x y}=\frac{K_{I}}{2 \sqrt{\pi a}}\left((r / 2 a)^{-1 / 2} \sin \frac{\theta}{2} \cos \frac{\theta}{2} \cos \frac{3 \theta}{2}-\right. \\
& \frac{3}{2}(r / 2 a)^{1 / 2} \sin \frac{\theta}{2} \cos ^{2} \frac{\theta}{2}-\sum_{n=1}^{\infty}(r / 2 a)^{n+\frac{1}{2}} C_{n}\left[\left(n+\frac{1}{2}\right) \sin \theta \cos \left(n-\frac{1}{2}\right) \theta\right] \\
& +\frac{K_{I I}}{2 \sqrt{\pi a}}\left((r / 2 a)^{\frac{-1}{2}} \cos \frac{\theta}{2}\left(1-\sin \frac{\theta}{2} \sin \frac{3 \theta}{2}\right)+\frac{3}{2}(r / 2 a)^{\frac{1}{2}} \cos \frac{\theta}{2}\left(1+\sin ^{2} \frac{\theta}{2}\right)+\right. \\
& \sum_{n=1}^{\infty}(r / 2 a)^{n+\frac{1}{2}} C_{n}\left[\cos \left(n+\frac{1}{2}\right) \theta-\left(n+\frac{1}{2}\right) \sin \theta \sin \left(n-\frac{1}{2}\right) \theta\right]
\end{aligned}
$$

where $\sigma_{\mathrm{xx}}, \sigma_{\mathrm{yy}}, \tau_{\mathrm{xy}}$ are the stress components and $r, \theta$ are the crack tip coordinate. The constant coefficients $C_{\mathrm{n}}$ were defined as:

$$
C_{n}=(-1)^{n} \frac{2 n+3}{2 n+2} \frac{1 \times 3 \times \ldots . \times(2 n-1)}{2 \times 4 \times \ldots .}
$$

Other parameters used in Eqs. (1) to (4) are defined as follows:

- $\quad a$ is the half length of center crack

- $\quad \beta$ is the angle between the crack and the $\mathrm{Y}$ axis shown in Figure 1

- $\quad r$ is the radial distance from crack tip

- $\quad \mathrm{n}$ is the number of terms after the first two terms of the Williams series expansion

- $\quad k$ is the ratio factor of loading in the $\mathrm{X}$ direction relative to the loading in the Y direction

- $\quad K_{\mathrm{I}}$ and $K_{\mathrm{II}}$ are the modes I and II stress intensity factors that are related to the singular terms of the stress series expansion

In the case of pure mode I loading, the load is applied perpendicular to the crack planes and caused them to open without any sliding. Mode II loading is related to shear loading and tends to slide one of the planes against the other one. The values of $K_{\mathrm{I}}$ and $K_{\mathrm{II}}$ are the main parameters for defining the state of crack tip stresses in different loading conditions. For a mixed-mode loading problem the superposition of $K_{\mathrm{I}}$ and $K_{\mathrm{II}}$ can be used for deriving the complex mixed mode stress field. For a 
general angled center crack plate subjected to biaxial loading the stress intensity factors can be determined from Papadopoulos (1993) as:

$$
\begin{aligned}
& K_{I}=\frac{\sigma_{\infty} \sqrt{\pi a}}{2}[(1+k)-(1-k) \cos 2 \beta], \\
& K_{I I}=\frac{\sigma_{\infty} \sqrt{\pi a}}{2}[(1-k) \sin 2 \beta] .
\end{aligned}
$$

Note that using the above relations and the stress relations of Mohr circle by taking into account only the first stress term (i.e. singular term), the crack tip stress field can be rewritten in the polar coordinate system as pointed out by Erdogan and Sih (1963):

$$
\begin{aligned}
\sigma_{\theta \theta} & =\frac{1}{\sqrt{2 \pi r}} \cos \frac{1}{2} \theta\left(K_{I} \cos ^{2} \frac{\theta}{2}-\frac{3}{2} K_{I I} \sin \theta\right), \\
\tau_{r \theta} & =\frac{1}{\sqrt{2 \pi r}} \cos \frac{1}{2} \theta\left(K_{I} \sin \theta+K_{I I}(3 \cos \theta-1)\right) .
\end{aligned}
$$

However, the effect of other higher order terms has been neglected in the tangential stress component provided in Eq. (7). But according to the extensive research studies (Williams \& Ewing 1972, Fett 2001, Kim \& Paulino 2003, Molla-abbasi \& Schutte 2008, Jogdand \& Murthy 2010, Aliha et al. 2010, Saghafi et al. 2010 Ayatollahi \& Aliha 2011, Ameri et al. 2012, Aliha et al. 2013, Zhou et al. 2013) the other terms of Williams series expansion may also have noticeable influences on the fracture behavior of cracked bodies. Hence in the upcoming section of this paper a more accurate tangential stress component is derived by considering the effects of higher order terms.

\section{Deriving a more accurate formulation for $\sigma_{\theta \theta}$}

By using a stress relationship of Mohr circle and considering the linear elastic fracture mechanics relations, $\sigma_{\theta \theta}$ could be derived in terms of $\sigma_{\mathrm{x}}, \sigma_{\mathrm{y}}$ and $\tau_{\mathrm{xy}}$ as below:

$$
\sigma_{\theta}=\left(\frac{\sigma_{x}+\sigma_{y}}{2}\right)-\left(\frac{\sigma_{x}-\sigma_{y}}{2}\right) \cos 2 \theta-\tau_{x y} \sin 2 \theta
$$

By replacing the Eqs. (1-3) into Eq. (9), and choosing the number of higher order terms, different relations can be derived for $\sigma_{\theta \theta}$. Accordingly, the influence of the first six terms in the Williams series expansion was taken into account in this paper to obtain the elastic tangential stress term. Since the obtained formulations were too long, the results of these calculations have not been provided here for the sake of brevity. In order to determine the maximum value of $\sigma_{\theta \theta}$ the obtain relations from Eq. (9) were maximized using the direct derivation of Eq. (9) in MATLAB code and the numerical values of $\sigma_{\theta \theta}$ were obtained for a large number of points. Based on the previous experimental works, including Maiti and Smith (1983) and Leevers et al. (1976), the direction of initial fracture from the crack plane is a figure between 0 and $\frac{-\pi}{2}$. Therefore this range was equally dividend to one hundred increments and the tangential stress values was calculated at each increment. Also, the MATLAB code was able to separate the stress terms of the Williams series given in relations (1), (2) and (3) and hence the influence of each term on the value of $\sigma_{\theta \theta}$ was computable. Consequently, the crack growth path of the investigated angled crack plate was obtained by connecting the locus of these calculated maximum tangential stress points for each crack inclination angle $\beta$.

\section{Results and discussion}

Calculations of this paper predict the initial direction of fracture growth $\theta_{\mathrm{c}}$ for each increment based on the maximum tangential stress. For this purpose, five different ratios of $r / a$ were chosen as: $(r / a=0.001,0.005,0.01,0.05,0.1)$ for each crack angle $(\beta)$. First for each $r / a$ the initial direction of crack growth $\left(\theta_{\mathrm{c}}\right)$ was calculated with only one term (or singular term) of stress series $\sigma_{\theta \theta}$. This calculation was then repeated by adding the other terms (i.e. the second to sixth terms) and for 
different angles of $(\beta)$ for the investigated cracked plate. Subsequently, the results of the predicted crack growth path were compared in Table 1with the experimental results reported by Maiti and Smith (1983). In Table 1, W =1 corresponds to the conventional conditions where only the singular term of stress is used. In the next five columns of Table 1 the calculated crack inclination angles have been presented based on the different number of terms used, specified by $\mathrm{W}=2,3,4,5,6$. For instance, $\mathrm{W}=2$ means the results of fracture initiation angle with sum of the first two terms of Williams series and $\mathrm{W}=6$ presents the results with the whole six terms.

\section{Table 1}

Initial crack growth path ( $\theta_{\mathrm{c}}$ in degree) calculated based on the maximum tangential stress criterion and by considering different terms of Williams series expansion.

\begin{tabular}{|c|c|c|c|c|c|c|c|c|}
\hline \multirow[t]{2}{*}{$\beta$ (degree) } & \multirow[t]{2}{*}{ r/a } & \multirow[t]{2}{*}{ Experimental data for $\left(-\theta_{\mathrm{c}}\right)$ (Maiti and Smith, 1983) } & \multicolumn{6}{|c|}{ Theoretical predictions for $\left(-\theta_{\mathrm{c}}\right)$} \\
\hline & & & $W=1$ & $W=2$ & $W=3$ & $W=4$ & $W=5$ & $W=6$ \\
\hline 15 & 0 & 68.8 & 65.4 & 68.8 & 68.8 & 68.8 & 68.8 & 68.8 \\
\hline 15 & 0.01 & 71.8 & 65.4 & 72.1 & 71.7 & 71.7 & 71.8 & 71.7 \\
\hline 15 & 0.01 & 73.4 & 65.4 & 74 & 73.3 & 73.3 & 73.4 & 73.4 \\
\hline 15 & 0.05 & 77 & 65.4 & 79 & 76.9 & 76.9 & 77 & 76.9 \\
\hline 15 & 0.1 & 77.8 & 65.4 & 81 & 77.8 & 77.8 & 77.8 & 77.8 \\
\hline 30 & 0 & 61.2 & 59.9 & 61.3 & 61.2 & 61.2 & 61.2 & 61.2 \\
\hline 30 & 0.01 & 62.6 & 59.9 & 62.9 & 62.5 & 62.5 & 62.5 & 62.5 \\
\hline 30 & 0.01 & 63.2 & 59.9 & 63.9 & 63.2 & 63.2 & 63.2 & 63.2 \\
\hline 30 & 0.05 & 64.6 & 59.9 & 68 & 64.6 & 64.6 & 64.6 & 64.6 \\
\hline 30 & 0.1 & 64.4 & 59.9 & 70.4 & 64.2 & 64.4 & 4.4 & 64.4 \\
\hline 45 & 0 & 53 & 53.1 & 53.1 & 53 & 53 & 53 & 53 \\
\hline 45 & 0.01 & 52.8 & 53.1 & 53.1 & 53.1 & 52.8 & 52.9 & 52.9 \\
\hline 45 & 0.01 & 52.6 & 53.1 & 53.1 & 52.9 & 52.5 & 52.5 & 52.5 \\
\hline 45 & 0.05 & 50.8 & 53.1 & 53.1 & 50.7 & 50.8 & 50.8 & 50.8 \\
\hline 45 & 0.1 & 49.2 & 53.1 & 53.1 & 48.9 & 49.1 & 49.1 & 49.1 \\
\hline 60 & 0 & 42 & 43.2 & 41.9 & 41.9 & 41.9 & 41.9 & 41.9 \\
\hline 60 & 0.01 & 40.4 & 43.2 & 40.4 & 40.3 & 40.3 & 40.3 & 40.3 \\
\hline 60 & 0.01 & 39.2 & 43.2 & 39.2 & 39.2 & 39.2 & 39.2 & 39.2 \\
\hline 60 & 0.05 & 35 & 43.2 & 34.7 & 35 & 35 & 35 & 35 \\
\hline 60 & 0.1 & 32.8 & 43.2 & 31.7 & 32.7 & 32.7 & 32.7 & 32.7 \\
\hline 75 & 0 & 24.8 & 26.7 & 24.7 & 24.8 & 24.8 & 24.8 & 24.8 \\
\hline 75 & 0.01 & 22.8 & 26.7 & 22.6 & 22.7 & 22.7 & 22.7 & 22.7 \\
\hline 75 & 0.01 & 21.4 & 26.7 & 21.2 & 21.5 & 21.5 & 21.5 & 21.5 \\
\hline 75 & 0.05 & 17.8 & 26.7 & 16.6 & 17.8 & 17.7 & 17.7 & 17.7 \\
\hline 75 & 0.1 & 16.2 & 26.7 & 14.2 & 16.3 & 16.2 & 16.2 & 16.3 \\
\hline
\end{tabular}

Maiti and Smith (1983) provided their experimental results up to the ratio $r / a=2$ for a brittle material with Poisson's ratio of 0.35 . Figs. 2 to 6 , compare the theoretical predicted crack growth paths based on the MTS criterion with the experimental results of Maiti and Smith (1983) obtained from the angled crack plate specimen. For each crack angle $(\beta)$ the experimental results have been compared with the theoretical paths with considering two different stress terms (i.e. $\mathrm{W}=1$ and $\mathrm{W}=6$ ). In these Figures the vertical axis shows the direction of fracture growth for each increment. It was observed that in general the theoretical results comply well with the experimental data when the sum of 6 terms of Williams series expansion is used. However, the good agreement between the experimental results and theoretical predictions was mostly observed for $r / a<1$ and for the higher $r / a$ ratios some discrepancies were seen between the experimental and theoretical results. A review of the results provided in Figs. 2 to 6 showed that in all mixed modes I/II conditions investigated for the angled crack plate subjected to biaxial tension, the predicted path using the higher number of terms of Williams series complies better with experimental results compared to using only the first singular term. The primary reason for this improvement in the prediction of initial crack growth path, can be attributed to the more accurate calculation of tangential stress component when the higher order terms of Williams series are used. It should be noted that only for a small region around the crack tip the singular terms of stress are dominant and for farther distances from the crack tip, the other terms may have significant effects on fracture behavior of the angled crack plate. Thus it can be seen from Figs. 2 to 6 that in the first increment of crack growth, there was no significant difference between the results of $\mathrm{W}=1$ and $\mathrm{W}=6$ cases, but by increasing the number of increments the deviation between the predicted values with one or six terms becomes more. 


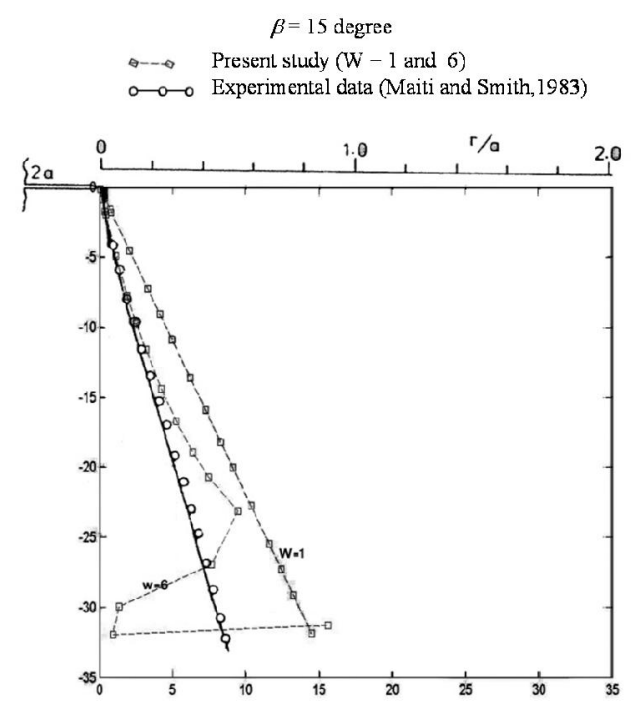

Fig. 2. Comparison of theoretical and experimental fracture paths obtained for $\beta=15^{\circ}$

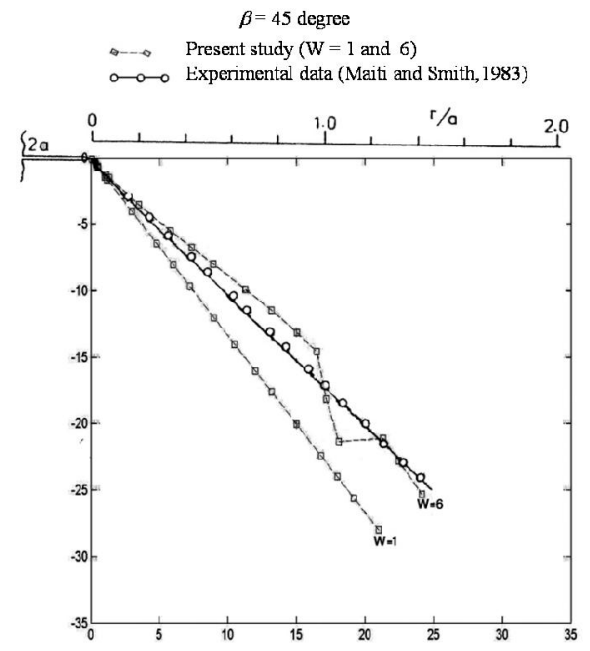

Fig. 4. Comparison of theoretical and experimental fracture paths obtained for $\beta=45^{\circ}$

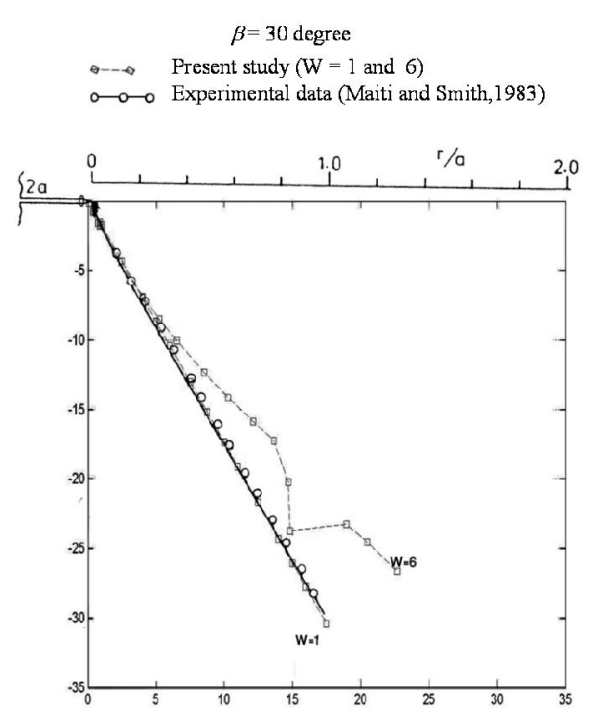

Fig. 3. Comparison of theoretical and experimental fracture paths obtained for $\beta$

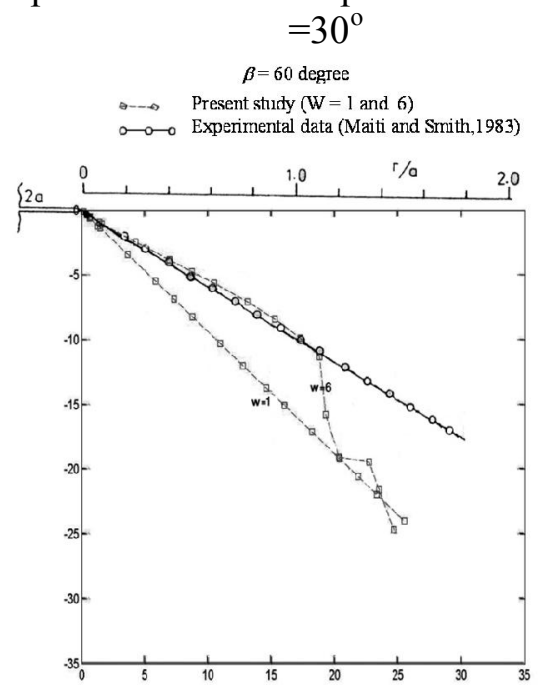

Fig. 5. Comparison of theoretical and experimental fracture paths obtained for $\beta=60^{\circ}$

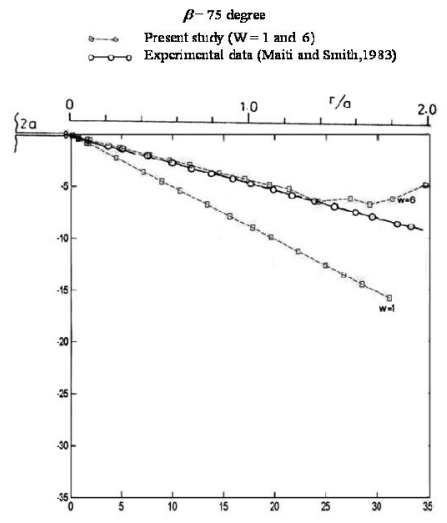

Fig. 6. Comparison of theoretical and experimental fracture paths obtained for $\beta=75^{\circ}$ 
Furthermore, as seen from Figs. 2 to 6 some discontinuities in the slope of the predicted paths were observed especially for the higher ratios of $r / a>1$ and for those cases where more than three terms of stress component were considered. These discontinuities could be due to numerically solving the problem and the high volume of complex mathematical and numerical calculations. A more important reason that could be noted for the discontinuities in the numerical results was related to the accuracy limitations of Eq. (4) to define the coefficients of the higher terms of the Williams series. Theocaris and Spyropoubs (1983) determined the coefficients $\left(C_{n}\right)$ in Eq. (4) by an experimental photoelastic method. Although this method was frequently used for experimental measuring and analyzing of stress in the cracked samples, the inherent errors are inevitable in the measurement and analysis of the experimental results. In particular, the errors are gradually increased for the higher terms of the Williams series. Therefore, the errors can be accumulated on the previously mentioned numerical errors, which explain the discontinuities in some parts of the predicted path. Another assumption that was used by Theocaris and Spyropoubs (1983) to derive the Eq. (4) was that the cracked plate dimensions are very large (i.e. infinite) in comparison with to the center crack dimensions. But since the photoelastic tests and brittle fracture experiments were performed on plate specimens with finite dimensions, the coefficients $\left(C_{\mathrm{n}}\right)$ might also contain other computational errors. In accordance with the above explanation, if an accurate method was available to determine the coefficients $\left(C_{\mathrm{n}}\right)$ in the investigated center cracked plate, a better numerical result could be obtained in the prediction of primary crack path, especially in the region where $r / a>1$.

\section{Conclusions}

In summary it can be stated that:

1. Mixed mode crack growth path of the angled center crack plate was predicted theoretically by the maximum tangential stress (MTS) criterion and by considering different terms of the Williams series expansion.

2. A more accurate description for the tangential stress component in the angled crack plate problem subjected to biaxial far field tension was obtained by taking into account the first six terms of Williams series expansion.

3. Good agreements were observed between the experimental fracture paths and theoretical fracture trajectory when the effect of higher order terms was also taken into account.

\section{References}

Aliha, M. R. M., \& Ayatollahi, M. R. (2009). Brittle fracture evaluation of a fine grain cement mortar in combined tensile-shear deformation. Fatigue \& Fracture of Engineering Materials \& Structures, 32(12), 987-994.

Aliha, M. R. M., Ayatollahi, M. R., Smith, D. J., \& Pavier, M. J. (2010). Geometry and size effects on fracture trajectory in a limestone rock under mixed mode loading. Engineering Fracture Mechanics, 77(11), 2200-2212.

Aliha, M. R. M., \& Ayatollahi, M. R. (2012). Analysis of fracture initiation angle in some cracked ceramics using the generalized maximum tangential stress criterion. International Journal of Solids and Structures, 49(13), 1877-1883.

Aliha, M. R. M., Hosseinpour, G. R., \& Ayatollahi, M. R. (2013). Application of Cracked Triangular Specimen Subjected to Three-Point Bending for Investigating Fracture Behavior of Rock Materials. Rock Mechanics and Rock Engineering, 1-12.

Aliha, M. R. M., \& Rezaei, M. (2011). Experimental and Theoretical Study of Fracture Paths in Brittle Cracked Materials Subjected to Pure Mode II Loading. Applied Mechanics and Materials, 70, 159-164.

Alpa, G., Bozzo, E., \& Gambarotta, L. (1980). Some observations on the path stability in fracture propagation for biaxially stressed plates. Engineering Fracture Mechanics, 13(4), 791-799. 
Ameri, M., Mansourian, A., Heidary Khavas, M., Aliha, M. R. M., \& Ayatollahi, M. R. (2011). Cracked asphalt pavement under traffic loading-A 3D finite element analysis. Engineering Fracture Mechanics, 78(8), 1817-1826.

Ayatollahi, M. R., Aliha, M. R. M., \& Hassani, M. M. (2006). Mixed mode brittle fracture in PMMA - an experimental study using SCB specimens. Materials Science and Engineering: A, 417(1), 348-356.

Ayatollahi, M. R., \& Aliha, M. R. M. (2009). Analysis of a new specimen for mixed mode fracture tests on brittle materials. Engineering Fracture Mechanics,76(11), 1563-1573.

Ayatollahi, M. R., Aliha, M. R. M., \& Saghafi, H. (2011). An improved semi-circular bend specimen for investigating mixed mode brittle fracture. Engineering Fracture Mechanics, 78(1), 110-123.

Erdogan, F., \& Sih, G. C. (1963). On the crack extension in plates under plane loading and transverse shear. Journal of Basic Engineering, 85, 519-527.

Fett, T. (2001). Stress intensity factors and T-stress for internally cracked circular disks under various boundary conditions. Engineering Fracture Mechanics, 68(9), 1119-1136.

Gómez, F. J., Elices, M., Berto, F., \& Lazzarin, P. (2009). Fracture of U-notched specimens under mixed mode: experimental results and numerical predictions. Engineering Fracture Mechanics, 76(2), 236-249.

Hussain, M. A., Pu, S. L., \& Underwood, J. (1974). Strain Energy Release Rate for a Crack Under Combined Mode I and Mode. Fracture analysis, 560, 1.

Jogdand, P. V., \& Murthy, K. S. R. K. (2010). A finite element based interior collocation method for the computation of stress intensity factors and T-stresses. Engineering Fracture Mechanics, 77(7), 1116-1127.

Kim, J. H., \& Paulino, G. H. (2003). T-stress, mixed-mode stress intensity factors, and crack initiation angles in functionally graded materials: a unified approach using the interaction integral method. Computer Methods in Applied Mechanics and Engineering, 192(11), 1463-1494.

Leevers, P. S., Radon, J. C., \& Culver, L. (1976). Fracture trajectories in a biaxially stressed plate. Journal of the Mechanics and Physics of Solids, 24(6), 381-395.

Maiti, S. K., \& Prasad, K. S. R. K. (1980). A study on the theories of unstable crack extension for the prediction of crack trajectories. International Journal of Solids and Structures, 16(6), 563-574.

Maiti, S. K., \& Smith, R. A. (1983). Comparison of the criteria for mixed mode brittle fracture based on the preinstability stress-strain field Part I: Slit and elliptical cracks under uniaxial tensile loading. International Journal of Fracture, 23(4), 281-295.

Molla-Abbasi, K., \& Schütte, H. (2008). On the full set of elastic T-stress terms of internal elliptical cracks under mixed-mode loading condition. Engineering Fracture Mechanics, 75(6), 1545-1568.

Saghafi, H., Ayatollahi, M. R., \& Sistaninia, M. (2010). A modified MTS criterion (MMTS) for mixed-mode fracture toughness assessment of brittle materials. Materials Science and Engineering: A, 527(21), 5624-5630.

Sih, G. C. (1974). Strain-energy-density factor applied to mixed mode crack problems. International Journal of Fracture, 10(3), 305-321.

Sumi, Y. (1985). Computational crack path prediction. Theoretical and applied fracture mechanics, 4(2), 149-156.

Theocaris, P. S., \& Spyropoulos, C. P. (1983). Photoelastic determination of complex stress intensity factors for slant cracks under biaxial loading with higher-order term effects. Acta Mechanica, 48(1-2), 57-70.

Papadopoulos, G. A. (1993). Fracture Mechanics. London, Springer-verlag Limited

Williams, M. L. (1957). On the stress distribution at the base of a stationary crack. Journal of Applied Mechanics, 24(1), 109-114.

Williams, J. G., \& Ewing, P. D. (1972). Fracture under complex stress - the angled crack problem. International Journal of Fracture Mechanics, 8(4), 441-446.

Zhou, Z., Xu, X., Leung, A. Y., \& Huang, Y. (2013). Stress intensity factors and T-stress for an edge interface crack by symplectic expansion. Engineering Fracture Mechanics, 102, 334-347. 